Historic, Archive Document

Do not assume content reflects current scientific knowledge, policies, or practices. 


\section{BIRDS OFTHE}

MONONGAHELA NATIONAL FOREST

CHECKLIST

Reserve aQL684

.W4B56 1996

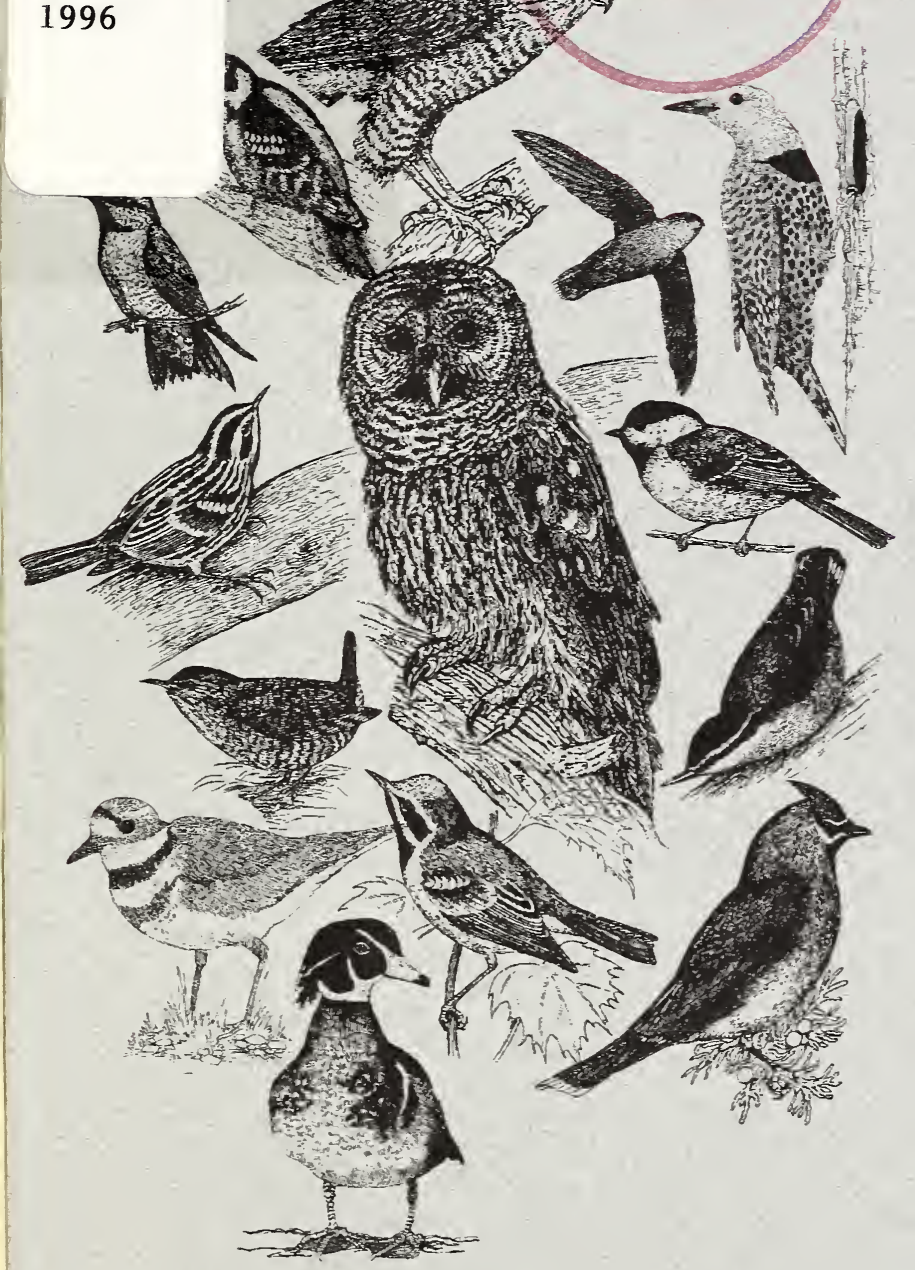

AMERICA'S
GREAT UNA S S S S
OUTDOORS 


\section{The Monongahela National Forest - over}

908,000 acres of diverse habitat used by 230 species of birds.

The Monongahela is located in the Appalachian Mountains of east central West Virginia at elevations of 1,000 to 4,861 feet. The forest stands of various tree species and age classes, and non-forested areas--such as wetlands, cliffs, grassy meadows, and cascading streams--all provide places for birds to feed, rest, and raise their young. This diverse landscape provides habitat for 70 species of resident birds, 89 breeding neotropical migrants, and 71 nonbreeding migratory bird species.

The Forest Service encourages you to try birding on the National Forest. Special efforts are taken to protect and provide the necessary habitats for birds--food -producing trees and shrubs are planted for food and cover; during timber harvest/firewood cutting, many cavity trees and snags are saved for nesting, feeding, and roosting sites. Through birding, you can become familiar with the Forest Service's role in protection and management which includes recreation, timber, wildlife, water, wilderness, and range resources.

This bird checklist was compiled to make your visit to the Monongahela National Forest more enjoyable, and to provide the opportunity for added wildlife appreciation.

Codes used in this bird checklist:

\section{$\underline{\text { Status }}$}

$\mathrm{R}=$ year-round resident of the Forest

$\mathrm{B}=$ breeds but leaves the area for the winter

$\mathrm{N}=$ nonbreeding spring and/or fall migrant (only stops on the Forest to rest and feed)

$\mathrm{V}=$ accidental or casual visitor (occasionally visits the area)

$\mathrm{W}=$ winter visitor to the Forest

\section{Habitat Frequented}

$\mathrm{O}=$ Open Areas (meadows, utility corridors, pasture)

$\mathrm{W}=$ Lakes, Ponds, Streams, Wetlands

$\mathrm{C}=$ Conifer Forest (Spruce, Pine, Hemlock, etc.)

$\mathrm{H}=$ Deciduous Forest (Oak, Maple, Beech, etc.)

$\mathrm{B}=$ Brush, Shrubs, Thickets

$\mathrm{E}=$ Forest Edge

Please send reports of any rare bird sightings (northern goshawk, cerulean warbler, bald eagle, peregrine falcon, and any species not appearing on this checklist) to: Forest Biologist, Monongahela National Forest, 200 Sycamore Street, Elkins, WV 26241-3962. Please include name, address, and phone number with date and location of sighting.

Illustrations of nuthatch, chickadee, wren, waxwing, swift, hummingbird, barred owl, black-and-white warbler, golden-winged warbler, downy woodpecker, and sharp-shinned hawk by Charles Joslin, Charlemont, Massachusetts. 


\section{LOONS (Gaviidae)}

Loon, Common

GREBES (Podicipedidae)

_Grebe, Horned

Grebe, Pied-billed

CORMORANTS (Phalacrocoracidae)

Cormorant, Double-crested

HERONS (Ardeidae)

Bittern. American

Egret, Cattle

N. W

Egret, Great

Heron, Great Blue

H..Heron, Green-backed

....Heron, Little Blue

\section{IBISES (Threskiornithidae)}

Ibis, White

$\begin{array}{ll}\mathrm{N} & \mathrm{W} \\ \mathrm{B}, \mathrm{W} & \mathrm{W}\end{array}$

N W

B W

N, V W

N, V $\quad$ W

B W

B $\quad W$

N, V W

SWANS, GEESE and DUCKS (Anatidae)

Bufflehead

Canvasback

Duck, American Black

Duck, Ring-necked

Duck, Ruddy

Duck, Wood

Gadwall

Goldeneye, Common

Goose, Canada

Goose, Snow

Mallard

Merganser, Common

Merganser, Hooded

Merganser, Red-breasted

Oldsquaw

Pintail, Northern

Redhead

Scaup, Lesser

Scoter, White-winged

Swan, Tundra

Teal, Blue-winged

Teal, Green-winged

Wigeon, American

$\mathrm{N}, \mathrm{V} \quad \mathrm{W}$

$\mathrm{N}=\mathrm{W}$

N W

B, W W

N W

N W

B, W W

N W

N W

$\mathrm{R} \quad \mathrm{O}, \mathrm{W}$

N W

B, W W

$\mathrm{N}=\mathrm{W}$

B W

N W

$\mathrm{N} \quad \mathrm{W}$

$\mathrm{N} \quad \mathrm{W}$

$\mathrm{N} \quad \mathrm{W}$

$\mathrm{N}=\mathrm{W}$

$\mathrm{N} \quad \mathrm{W}$

N W

N W

B W

N W

VULTURES (Cathartidae)

Vulture, Black

B O,C,H,E

B, W O,C,H,E

HAWKS and EAGLES (Accipitridae)

Eagle, Bald

Eagle, Golden

B

$\mathrm{O}, \mathrm{C}, \mathrm{H}, \mathrm{W}$

Eagle, Bald

N

B $\quad \mathrm{O}, \mathrm{C}, \mathrm{H}, \mathrm{W}$

Eagle, Golden

N

R C,H

Goshawk, Northern 
Harrier, Northern

$\begin{array}{ll}\text { B,W } & \text { O,W } \\ \text { B } & \text { H } \\ \text { R } & \text { C,H,E } \\ \text { B,W } & \text { H,E } \\ \text { B,W } & \text { O,H,E } \\ \text { N,W } & \\ \text { R } & \text { C,H,E } \\ \text { N } & \text { W }\end{array}$

\section{FALCONS (Falconidae)}

Falcon, Peregrine

$\begin{array}{ll}\text { B } & \text { O,H } \\ \mathrm{R} & \mathrm{O}, \mathrm{E}\end{array}$

Kestrel, American

Merlin

$\mathrm{N}$

QUAIL, GROUSE and TURKEYS (Phasianidae)

Bobwhite, Northern

Grouse, Ruffed

$\begin{array}{ll}R & O, B, E \\ R & \text { C,H,B,E } \\ R & \text { O,C,H,E }\end{array}$

-Turkey, Wild

$\mathrm{O}, \mathrm{C}, \mathrm{H}, \mathrm{E}$

RAILS, GALLINULES and COOTS (Rallidae)

_Coot, American

Moorhen, Common

_Rail, Virginia

Sora

$\mathrm{N} \quad \mathrm{W}$

$\mathrm{N} \quad \mathrm{W}$

N W

CRANES (Gruidae)

Crane, Sandhill

\section{PLOVERS (Charadriidae)}

Killdeer

Plover, Black-bellied

Plover, Lesser Golden

Plover, Semipalmated

SANDPIPERS (Scolopacidae)

Sandpiper, Least

Sandpiper, Semipalmated

Sandpiper, Solitary

Sandpiper, Spotted

Sandpiper, Upland

Snipe, Common

_Woodcock, American

Yellowlegs, Greater

Yellowlegs, Lesser

SKUAS, GULLS and TERNS (Laridae)

_Gull, Bonaparte's

_Gull, Herring

Gull, Ring-billed

PIGEONS and DOVES (Columbidae)

Dove, Mourning

Dove, Rock

CUCKOOS (Cuculidae)

_Cuckoo, Black-billed

$\mathrm{N} \quad \mathrm{W}$

V

W

B O,W

$\mathrm{N} \quad \mathrm{W}$

$\mathrm{N} \quad \mathrm{W}$

$\mathrm{N} \quad \mathrm{W}$

$\mathrm{N} \quad \mathrm{W}$

$\mathrm{N} \quad \mathrm{W}$

$\mathrm{N} \quad \mathrm{W}$

B $\quad$ W

N W

B,W W

$\mathrm{B}, \mathrm{W} \quad \mathrm{O}, \mathrm{W}, \mathrm{B}, \mathrm{E}$

$\mathrm{N} \quad \mathrm{W}$

N W

$\mathrm{N} \quad \mathrm{W}$

$\mathrm{N} \quad \mathrm{W}$

$\mathrm{N} \quad \mathrm{W}$

B,W O,B,E

$\mathrm{R} \quad \mathrm{O}$

B $\quad \mathrm{H}, \mathrm{B}, \mathrm{E}$

B $\mathrm{H}, \mathrm{B}, \mathrm{E}$ 


\section{BARN OWLS (Tytonidae)}

Barn-Owl, Common

$\mathrm{R}$

O,E

TYPICAL OWLS (Strigidae)

_Owl, Barred

$\begin{array}{ll}\text { R } & \mathrm{C}, \mathrm{H} \\ \mathrm{R} & \mathrm{C}, \mathrm{H}, \mathrm{E} \\ \mathrm{R} & \mathrm{C}, \mathrm{H} \\ \mathrm{R} & \mathrm{C}, \mathrm{H} \\ \mathrm{V} & \\ \mathrm{V} & \\ \mathrm{R} & \mathrm{O}, \mathrm{C}, \mathrm{H}, \mathrm{E}\end{array}$

_Owl, Great Horned

_Owl, Long-eared

Owl, Northern Saw-whet

_Owl, Short-eared

Owl, Snowy

Screech-Owl, Eastern

GOATSUCKERS (Caprimulgidae)

_Nighthawk, Common

Whippoorwill

SWIFTS (Apodidae)

Swift, Chimney

HUMMINGBIRDS (Trochilidae)

_Hummingbird, Ruby-throated

Hummingbird, Rufous

KINGFISHERS (Alcedinidae)

Kingfisher, Belted

\section{WOODPECKERS (Picidae)}

Flicker, Northern

Sapsucker, Yellow-bellied

Woodpecker, Downy

Woodpecker, Hairy

_.Woodpecker, Pileated

__.Woodpecker, Red-bellied

Woodpecker, Red-headed

TYRANT FLYCATCHERS (Tyrannidae)

Flycatcher, Acadian

Flycatcher, Alder

Flycatcher, Great-Crested

Flycatcher, Least

Flycatcher, Olive-sided

Flycatcher, Willow

Flycatcher, Yellow-bellied

Kingbird, Eastern

- Phoebe, Eastern

Wood-Pewee, Eastern

\section{LARKS (Alaudidae)}

Lark, Horned

SWALLOWS (Hirundinidae)

_Swallow, Bank

B

B

$\mathrm{O}$

B

$\mathrm{O}$

B

$\mathrm{O}, \mathrm{H}, \mathrm{E}$

V

$\mathrm{B}, \mathrm{W} \quad \mathrm{W}$

$\begin{array}{ll}\text { B,W } & \text { O,C,H,E } \\ \text { B,W } & \text { C,H } \\ \text { R } & \text { C,H,E } \\ \text { R } & \text { C,H,E } \\ \text { R } & \text { C,H } \\ \text { R } & \text { C,H,E } \\ \text { R } & \text { O,H,E }\end{array}$

__Swallow, Barn

B

$\mathrm{H}$

B $\mathrm{O}, \mathrm{B}, \mathrm{E}$

B $\mathrm{H}, \mathrm{E}$

B $\quad \mathrm{H}, \mathrm{E}$

B C,H,E

B $\quad \mathrm{O}, \mathrm{B}, \mathrm{E}$

B $\quad$ C,E

B O,E

B O,E

B C,H,E

Swallow, Cliff

S..Swallow, Northern Rough-winged

_Swallow, Tree

$\mathrm{R}$

$\mathrm{O}$

B O,W

B $\quad O$

B O

B $\quad \mathrm{O}$

B O 
JAYS and CROWS (Corvidae)

_Crow, American

Jay, Blue

$\mathrm{O}, \mathrm{C}, \mathrm{H}, \mathrm{E}$

Raven, Common $\mathrm{O}, \mathrm{C}, \mathrm{H}, \mathrm{B}, \mathrm{E}$

$\mathrm{O}, \mathrm{C}, \mathrm{H}, \mathrm{E}$

CHICKADEES and TITMICE (Paridae)

Chickadee, Black-capped

Chickadee, Carolina

Titmouse, Tufted

NU'THATCHES (Sittidae)

Nuthatch, Red-breasted

Nuthatch, White-breasted

CREEPERS (Certhiidae)

Creeper, Brown

WRENS (Troglodytidae)

_.Wren, Bewick's

Wren, Carolina

_Wren, House

_-Wren, Marsh

_Wren, Sedge

Wren, Winter

THRUSHES (Muscicapidae)

_Bluebird, Eastern

Gnatcatcher, Blue-gray

Kinglet, Golden-crowned

Kinglet, Ruby-crowned

Robin, American

Thrush, Gray-cheeked

-.Thrush, Hermit

Thrush, Swainson's

Thrush, Wood

Veery

$\begin{array}{ll}\mathrm{R} & \mathrm{O}, \mathrm{C}, \mathrm{H}, \mathrm{E} \\ \mathrm{R} & \mathrm{O}, \mathrm{C}, \mathrm{H}, \mathrm{B}, \mathrm{E} \\ \mathrm{R} & \mathrm{O}, \mathrm{C}, \mathrm{H}, \mathrm{E}\end{array}$

$\mathrm{R} \quad \mathrm{O}, \mathrm{C}, \mathrm{H}, \mathrm{B}, \mathrm{E}$

$\mathrm{R} \quad \mathrm{C}, \mathrm{H}, \mathrm{B}, \mathrm{E}$

R. $\quad \mathrm{C}, \mathrm{H}, \mathrm{E}$

R C

$\mathrm{R} \quad \mathrm{C}, \mathrm{H}, \mathrm{E}$

$\mathrm{B}, \mathrm{W} \quad \mathrm{C}, \mathrm{H}$

B O,B,E

R O,B,E

B $\mathrm{O}, \mathrm{H}, \mathrm{B}, \mathrm{E}$

V W

B $\quad$ W

$\mathrm{B}, \mathrm{W} \quad \mathrm{C}, \mathrm{H}, \mathrm{B}$

MOCKINGBIRDS and THRASHERS (Mimidae)

Catbird, Gray

Mockingbird, Northern

Thrasher, Brown

PIPITS and WAGTAILS (Motacillidae)

Pipit, American

WAXWINGS (Bombycillidae)

Waxwing, Cedar

B,W O,E

B H,B,E

$\mathrm{B}, \mathrm{W} \quad \mathrm{C}$

N

B O,C,H,B,E

$\mathrm{N}$

B $\mathrm{C}, \mathrm{H}$

B C

B C,H

B $\mathrm{C}, \mathrm{H}, \mathrm{B}$

STARLINGS (Sturnidae)

Starling, European

VIREOS (Vireonidae)

_._Vireo, Philadelphia

Vireo, Red-eyed

_Vireo, Solitary

B B,E

R O,B,E

B B,E

_._Vireo, Warbling

Vireo, White-eyed

N

B

C,H,B,E

_Vireo, Yellow-throated

$\mathrm{R}$

O,E

$\mathrm{N}$

B $\mathrm{H}$

B C,H

B $\mathrm{C}, \mathrm{H}$

B $\mathrm{H}, \mathrm{B}, \mathrm{E}$

B $\mathrm{H}$ 


\section{WOOD WARBLERS, TANAGERS, SPARROWS and BLACKBIRDS}

(Emberizidae)

\section{Warblers}

Chat, Yellow-breasted

Ovenbird

Parula, Northern

Redstart, American

Warbler, Bay-breasted

Warbler, Black-and-White

Warbler, Blackburnian

Warbler, Blackpoll

Warbler, Black-throated Blue

Warbler, Black-throated Green

Warbler, Blue-winged

Warbler, Canada

Warbler, Cape May

Warbler, Cerulean

Warbler, Chestnut-sided

Warbler, Connecticut

_Warbler, Golden-winged

Warbler, Hooded

__Warbler, Kentucky

Warbler, Magnolia

Warbler, Mourning

Warbler, Nashville

Warbler, Orange-crowned

Warbler, Palm

Warbler, Pine

Warbler, Prairie

Warbler, Prothonotary

_Warbler, Swainson's

_Warbler, Tennessee

_Warbler, Wilson's

Warbler, Worm-eating

Warbler, Yellow

Warbler, Yellow-rumped

Warbler, Yellow-throated

Waterthrush, Northern

Waterthrush, Louisiana

Yellowthroat, Common

Tanagers

_Tanager, Scarlet

N

B

B

Tanager, Summer

Grosbeaks, Buntings, Tanagers and Sparrows

Bunting, Indigo

Bunting, Snow

Cardinal, Northern

Grosbeak, Rose-breasted

Junco, Dark-eyed

_Sparrow, American Tree
B

B

B

B

$\mathrm{N}$

B

B

N

B

B

B

B

N

B

B

B

B

B

B

B

B

$\mathrm{N}$

N

B

B

$\mathrm{N}$

B

N

N

B

B

B

B

B

B

B

O,B,E

C, $\mathrm{H}$

C, $\mathrm{H}$

H,B,E

$\mathrm{H}$

C,H

C, H

$\mathrm{C}, \mathrm{H}$

H,B,E

$\mathrm{C}, \mathrm{H}, \mathrm{B}, \mathrm{E}$

H

B,E

$\mathrm{O}, \mathrm{B}, \mathrm{E}$

$\mathrm{H}, \mathrm{E}$

$\mathrm{H}$

C,B,E

$\mathrm{B}, \mathrm{E}$

C,H,B,E

C

O,B

C, H

$\mathrm{H}$

$\mathrm{B}, \mathrm{E}$

C,E

C, $\mathrm{H}$

W

W

O,B,E

C,H

H

B,E

H,B,E

$\mathrm{H}, \mathrm{B}, \mathrm{E}$

B

B,W

$\mathrm{O}, \mathrm{C}, \mathrm{H}, \mathrm{B}, \mathrm{E}$ 
_Sparrow, Chipping

Sparrow, Field

Sparrow, Fox

Sparrow, Grasshopper

Sparrow, Henslow's

Sparrow, Lark

__Sparrow, Savannah

Sparrow, Song

Sparrow, Vesper

Sparrow, Lincoln's

Sparrow, Swamp

Sparrow, White-crowned Sparrow, White-throated Sparrow, Rufous-sided

\section{Blackbirds and Orioles}

\section{Bobolink}

Blackbird, Red-winged Blackbird, Rusty

Cowbird, Brown-headed

Grackle, Common

Meadowlark, Eastern

_Oriole, Orchard

Oriole, Northern

FINCHES (Fringillidae)

Crossbill, Red

Crossbill, White-winged

_Finch, Purple

Finch, House

_Goldfinch, American

__rosbeak, Evening

_Grosbeak, Pine

Redpoll, Common

Siskin, Pine

WEAVERS (Passeridae)

__Sparrow, House

$\begin{array}{ll}\text { B } & \text { O,H,E } \\ \text { B,W } & \text { O,B,E } \\ \text { N } & \\ \text { B } & O \\ \text { B } & \text { O } \\ \text { B } & \text { O } \\ \text { B } & \text { O } \\ \text { R } & \text { O,B,E } \\ \text { B } & \text { O,E } \\ \text { N } & \\ \text { B,W } & \text { W } \\ \text { N } & \\ \text { B,W } & \text { C,H,B,E } \\ \text { B,W } & \text { O,B,E }\end{array}$

B $\quad \mathrm{O}$

B O,W

$\mathrm{N}$

B O,C,H,E

B $O$

$\mathrm{B}, \mathrm{W} \quad \mathrm{O}$

B $\quad$ O,E

B $\mathrm{O}, \mathrm{H}, \mathrm{E}$

B,W C

$\mathrm{N}$

$\mathrm{B}, \mathrm{W} \quad \mathrm{C}, \mathrm{E}$

R O

$\mathrm{B}, \mathrm{W} \quad \mathrm{O}, \mathrm{E}$

W

$\mathrm{N}$

W

B $\quad$ C

$\mathrm{R}$

The USDA Forest Service is a multicultural organization and committed to the goal of ensuring equal opportunity for all in employment and program delivery.

\section{This brochure is available in large} print.

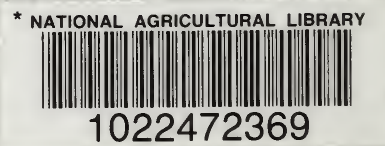

